Tropical Journal of Pharmaceutical Research July 2016; 15 (7): 1401-1411

ISSN: 1596-5996 (print); 1596-9827 (electronic)

(C) Pharmacotherapy Group, Faculty of Pharmacy, University of Benin, Benin City, 300001 Nigeria.

All rights reserved.

Available online at http://www.tjpr.org

Original Research Article

http://dx.doi.org/10.4314/tjpr.v15i7.8

\title{
Design, synthesis and antiproliferative activity of hydroxyacetamide derivatives against HeLa cervical carcinoma cell and breast cancer cell line
}

\author{
Supriyo Saha ${ }^{1 *}$, Dilipkumar $\mathrm{Pal}^{2}$ and Sushil Kumar ${ }^{3}$ \\ ${ }_{1}^{1}$ Department of Pharmaceutical Sciences, Sardar Bhagwan Singh Post Graduate Institute of Biomedical Sciences and \\ Research, Dehradun-248001, ${ }^{2}$ Department of Pharmaceutical Sciences, Division of Pharmaceutical Chemistry, Guru \\ Ghasidash Vishwavidyalaya, Koni, Bilashpur, Chhattisgarh, ${ }^{3}$ Faculty of Pharmacy, IFTM University, Moradabad, India
}

*For correspondence: Email: supriyo9@gmail.com; Tel: 7895424583; 0135-6457512; Fax: 0135-2733038

\begin{abstract}
Purpose: To design and develop a new series of histone deacetylase inhibitors (FP1 - FP12) and evaluate their inhibitory activity against hydroxyacetamide (HDAC) enzyme mixture-derived HeLa cervical carcinoma cell and MCF-7.

Methods: The designed molecules (FP1 - FP12) were docked using AUTODOCK 1.4.6. FP3 and FP8 showed higher interaction comparable to the prototypical HDACl. The designed series of 2-[[(3Phenyl/substituted Phenyl-[4-\{(4-(substituted phenyl)ethylidine-2-Phenyl-1,3-Imidazol-5-One\}](-4H1,2,4-triazol-5-yl)sulfanyl]-N-hydroxyacetamide derivatives (FP1-FP12) was synthesized by merging 2[(4-amino-3-phenyl-4H- 1, 2, 4-triazol-5-yl) sulfanyl]-N-hydroxyacetamide and 2-\{[4-amino-3-(2hydroxyphenyl)-4H-1,2, 4-triazol-5-yl]sulfanyl[3-N hydroxyacetamide derivatives with aromatic substituted oxazolone. The biological activity of the synthesized molecule (FP1-FP12) was evaluated against HDAC enzyme mixture-derived HeLa cervical carcinoma cell and breast cancer cell line (MCF-7).

Results: HDAC inhibitory activity of FP10 showed higher $I_{50}$ (half-maximal concentration inhibitory activity) of $0.09 \mu \mathrm{M}$, whereas standard SAHA molecule showed $I_{50}$ of $0.057 \mu \mathrm{M}$. On the other hand, FP9 exhibited higher $\mathrm{GI}_{50}$ (50\% of maximal concentration that inhibited cell proliferation) of $22.8 \mu \mathrm{M}$ against MCF-7 cell line, compared with the standard, adriamycin, with $\mathrm{GI}_{50}$ of (-) $50.2 \mu \mathrm{M}$.

Conclusion: Synthesis, spectral characterization, and evaluation of HDAC inhibition activity and in vitro anticancer evaluation of novel hydroxyacetamide derivatives against MCF-7 cell line have been achieved. The findings indicate the emergence of potentialanticancer compounds.
\end{abstract}

Keywords: Molecular docking, Hydroxyacetamide derivative, Histone deacetylase inhibition activity, MCF-7 cell line

Tropical Journal of Pharmaceutical Research is indexed by Science Citation Index (SciSearch), Scopus, International Pharmaceutical Abstract, Chemical Abstracts, Embase, Index Copernicus, EBSCO, African Index Medicus, JournalSeek, Journal Citation Reports/Science Edition, Directory of Open Access Journals (DOAJ), African Journal Online, Bioline International, Open-J-Gate and Pharmacy Abstracts

\section{INTRODUCTION}

Histone deacetylase (HDAC) enzyme is one of the leading targets in the process of anticancer drug development. HDAC was divided into three distinct structural classes as class (I/II) zincdependent and class (111) NAD dependent [1]. These enzymes are a part of multiprotein complexes, catalyzing the removal of acetyl group from lysine residue on protein including histone. Suberoyl anilide hydroxamic acid was the primary important histone deacetylase inhibitor (HDACl) (Figure 1).

Suberoyl anilide hydroxamic acid has three basic parts as surface recognition, linker and metal 
binding portions. HDAC inhibitors have shown to bind the active site and block the substrate access, causing a resultant accumulation of acetylated histones [2]. HDACls inhibit tumor growth, cell differentiation and programmed cell death. HDACls induce cancer cell cycle arrest, growth inhibition, differentiation, and programmed cell death [3]. $\mathrm{HDACl}$ induced cell cycle arrest and growth inhibition is usually correlated with transcriptional activation of p21WAF1/CIP1, p27KIP1, GADD45a [4] and inhibition of cyclin $A$, cyclin $D$ and thymidylate synthetase [5]. The most important HDACl are the hydroxamic acids group followed by the benzamides, the cyclic tetra peptides, the carboxylic acids and the electrophilic ketones [6]. These observations prompted us to modify the linker portion to an imidazolo triazolated moiety and enacting the metal binding hydroxamic acid portion and molecular docking against 1T69, synthesize and check their in vitro HDAC inhibition, antiproliferative efficacy against MCF-7 cell line.<smiles>O=C(CCCCCCC(=O)Nc1ccccc1)NO</smiles>

\section{Surface Recognition Linker Metal Binding}

Figure 1: Structure of basic pharmacophore of SAHA and synthesized HDAC inhibitor

\section{EXPERIMENTAL}

\section{Molecular docking protocol and binding analysis}

All computational studies were carried out using AUTODOCK 4.0.1. The geometry of HDAC-8 was extracted from the Brookhaven protein data bank (PDB entry code: 1T69) complexes with the irreversible inhibitor SAHA (Suberoyl Anilde Hydroxamic acid). All the residues within $20 \AA$ core from SAHA were used to define the metal binding site. For the docking, a grid spacing of $0.375 \AA$ and $126 \times 90 \times 90$ number of points was used. The grid was centered on the mass center of the experimental bound SAHA coordinates. Autodock generated 10 possible binding conformations. A default protocol was applied, with an initial population of 150 randomly placed individuals, a maximum number of $2.5 \times 10^{5}$ energy evaluations, and a maximum number of $2.7 \times 10^{4}$ generations. A mutation rate of 0.02 and a crossover rate of 0.8 were used [7-10]. Cluster analysis of the docking results using root mean square deviation (RMSD) tolerance of $2 \AA$.

\section{Materials and equipment}

Melting points were checked using open capillary method Veego Electronics Apparatus. The IR spectra for comparison of synthesized compounds were recorded on a Perkin Elmer (serial no: 78625) FTIR spectrophotometer. HNMR spectra were recorded on Bruker Avance DRX300 300MHz FTNMR spectrometer using DMSO-d6 as solvent. The chemical shifts were measured at $\delta$ units (reported as ppm) relative to TMS and signals are reported as s (singlet), d (doublet), t (triplet), q (quartet, m (multiple). Mass spectra were also recorded. All 1HNMR and Mass spectra was done by IICB, Kolkata and CDRI, Luck now. UV spectroscopy was done by the help of Shimadzu UV-1700 Spectroscopy. Elemental analysis was performed using a microanalytical unit. All chemicals were procured from sigma Aldrich and Merck. All the reactions were routinely checked by precoated Merck thin layer chromatographic plate using toluene:methanol $(9: 1)$ as solvent system.

\section{Chemistry}

\section{Synthesis of 2-chloro-N-hydroxyacetamide (III)}

Methanol $(12 \mathrm{~mL})$ and $0.0336 \mathrm{M}, 2.34 \mathrm{~g}$ of hydroxylamine hydrochloride were placed over a heated magnetic plate in a flask. The mixture was stirred for $5 \mathrm{~min}$ and added drop wise to a previously prepared methanol solution of 0.0501 $\mathrm{M}, 2.81 \mathrm{~g}$ potassium hydroxide. Then the resulting solution was cooled to room temperature and filtered. The filtrate was stored and used for the next step.

Then $25 \mathrm{ml}$ of methanol was taken in a $250 \mathrm{ml}$ beaker and stir over magnetic stirrer. Total synthetic setup was done under fuming cupboard. Pour $0.07 \mathrm{M}, 5.6 \mathrm{ml}$ of chloroacetyl chloride from dropping funnel onto the methanol and mixed it properly. Then hydroxylamine stock solution was added dropwise to the choloroacetyl chloride and stir for $2 \mathrm{~h}$. The product was obtained by filtration. Filtrate was washed with diethyl ether and recrystallized from methanol.

Physical Nature: White Colored powder solid. Yield: $33.25 \%$ M.p $=138{ }^{\circ} \mathrm{C}$. Total synthetic procedure was report in Scheme 1.

General procedure for the preparation of 2[(4-amino-3-phenyl/3-(2-hydroxy phenyl)-4H- 
1, 2, 4-Triazol-5-yl) sulfanyl]-N-hydroxyacetamide (IX and XV)

4-amino-3-phenyl-4H-1, 2, 4-triazole-5-thiol (VIII) was synthesized from benzoic acid and 2-(4amino-5-sulfanyl-4H-1, 2, 4-triazol-3-yl) phenol (XIV) from salicylic acid by adopting Reid Hindel Process. Both 4-amino-3-phenyl-4H-1, 2, 4- triazole-5-thiol (VIII) and 2-(4-amino-5-sulfanyl$4 \mathrm{H}-1,2$, 4-triazol-3-yl) phenol (XIV) were stirred with 2-chloro-N-hydroxyacetamide (III) in dimethyl formamide (DMF) solution for $1 \mathrm{~h}$ respectively to achieve compound $\mathbf{I X}$ and $\mathbf{X V}$. The general procedure for the synthesis of IX and $\mathbf{X V}$ was reported in Scheme 2 and 3.<smiles>C[13CH]=C(Cl)C(=O)CCl</smiles>

Scheme 1: (a) Stir 2 h (33.25 \%)<smiles>CCC(=O)c1ccc(-c2nnc(S)n2N)cc1C(=O)NNC(=O)CCl</smiles>

Scheme 2: (a) Conc $\mathrm{H}_{2} \mathrm{SO}_{4}, \mathrm{EtOH}, 80^{\circ} \mathrm{C}, 4 \mathrm{~h}$ ( 47.05\%): (b) $\mathrm{NH}_{2} \mathrm{NH}_{2} \cdot \mathrm{H}_{2} \mathrm{O}$, EtOH, $120^{\circ} \mathrm{C}, 6 \mathrm{~h}(31.01 \%$ ): (c) $\mathrm{CS}_{2}, \mathrm{KOH}, \mathrm{EtOH}$, Stir $15 \mathrm{~h}(44.42 \%):\left(\right.$ d) $\mathrm{NH}_{2} \mathrm{NH}_{2} \cdot \mathrm{H}_{2} \mathrm{O}, 130^{\circ} \mathrm{C}, 6 \mathrm{~h}$ (94.64\%): (f) DMF, Stir, $1 \mathrm{H}(45.20 \%)$

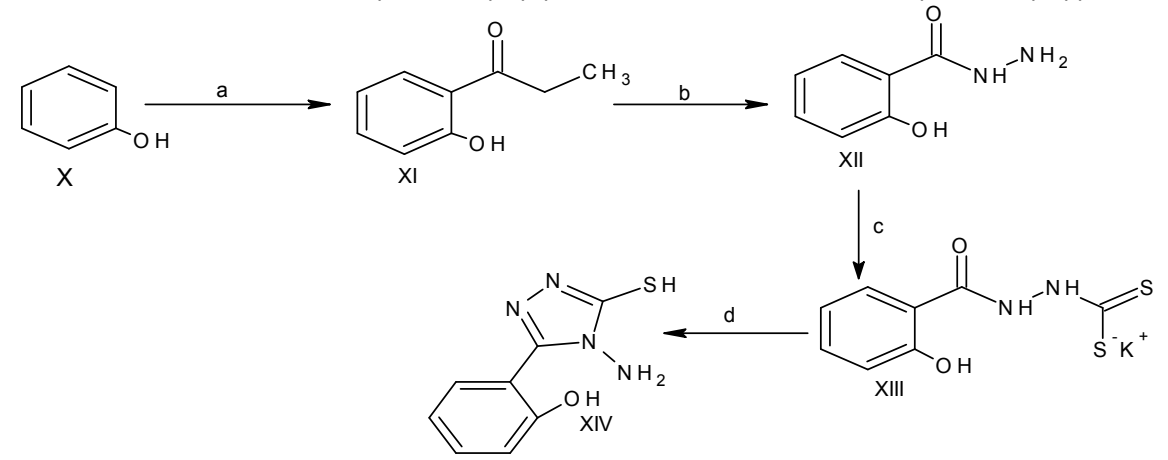<smiles>Cc1ccccc1-c1nnc(SCC(=O)NO)n1N</smiles>

Scheme 3: (a) Concentrated $\mathrm{H}_{2} \mathrm{SO}_{4}, \mathrm{EtOH}, 80^{\circ} \mathrm{C}, 4 \mathrm{~h}$ ( $49.25 \%$ ): (b) $\mathrm{NH}_{2} \mathrm{NH}_{2} \cdot \mathrm{H}_{2} \mathrm{O}$, EtOH, $120^{\circ} \mathrm{C}, 6 \mathrm{~h}$ (28.35\%): (c)

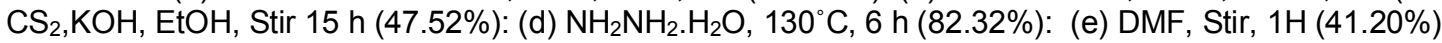


General procedure for the preparation of (4E)4-[(2-) methylidene]-2-phenyl-1,3-oxazol$5(4 \mathrm{H})$-one (XVIIla-f)

An amount of $0.022 \mathrm{M}$ substituted aromatic aldehyde (benzaldehyde, salicylaldehyde, 4methoxy benzaldehyde, anisaldehyde, furfuraldehyde, cinnamaldehyde), $1.8 \mathrm{~g}$ of 0.022 $\mathrm{M}$ sodium acetate and $4.0 \mathrm{~g}$ of $0.022 \mathrm{M}$ hippuric acid were suspended in $0.066 \mathrm{M}$ glacial acetic acid solution. The mixture was refluxed for $2 \mathrm{~h}$ under water bath.

After cooling the mixture, add $10 \mathrm{~mL}$ of ethanol into the solution and kept for overnight at below 5 ${ }^{\circ} \mathrm{C}$. The obtained precipitation was filtered and washed the filter cake with ethanol followed by drying under vacuum. Synthesis was performed as per Scheme 4 and result reported in Table 2.

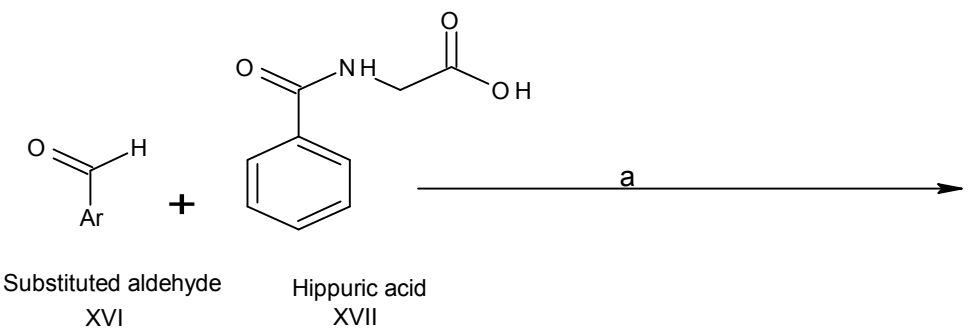

General procedure for the preparation of 2[[(3- substituted phenyl-[4-\{(4-(substituted phenyl) ethylidine-2-Phenyl-1,3-Imidazol-5One\}](-4H-1,2,4-triazol-5-yl)sulfanyl]-Nhydroxyacetamide (FP1 - 12)

Equimolar concentration (0.01 M) of (4E)-4[(substituted phenyl/methyl) methylidene]-2phenyl-1,3-oxazol-5(4H)-one and $0.01 \mathrm{M}$ of 2-[(4amino-3-phenyl-4H- 1, 2, 4-triazol-5-yl) sulfanyl]$\mathrm{N}$-hydroxyacetamide/2-\{[4-amino-3-(2-hydroxy phenyl)-4H-1, 2, 4-triazol-5-yl]sulfanyl\}-Nhydroxyacetamide was added to a $250 \mathrm{~mL}$ RBF and refluxed for $5 \mathrm{~h}$ at $150{ }^{\circ} \mathrm{C}$ under oil bath using $0.01 \mathrm{M}$ pyridine and $0.01 \mathrm{M}$ Zeolite $(\mathrm{Y}-\mathrm{H})$ as catalyst. After completion of the reaction, the excess of pyridine was distilled off. The resultant solution was cooled and poured into crushed ice and hydrochloric acid mixture. The obtained product was filtered and recrystallized from ethanol. Total synthetic procedure from FP1-12 was reported in Schemes 5 and 6.

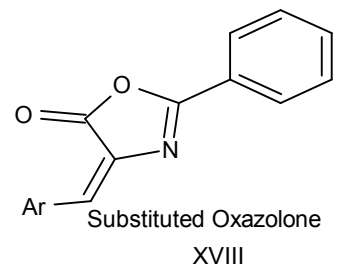

Scheme 4: (g) $\mathrm{CH}_{3} \mathrm{COONa}, \mathrm{CH}_{3} \mathrm{COOH}, 2$ h (XVIIla. $52.12 \%$, XVIIllb. $55.13 \%$, XVIIIc. $69.13 \%$, XVIIId. $63.18 \%$, XVIIIle. $48.19 \%$, XVIIIf. $64.85 \%)$

Table 2: Reaction parameters for compounds XVIII (a-f)

\begin{tabular}{|c|c|c|c|}
\hline Compound & Yield (\%) & $\begin{array}{c}\text { Melting } \\
\text { point ( }\left({ }^{\circ} \mathrm{C}\right)\end{array}$ & FTIR data $\left((\mathrm{KBr}) \mathrm{v} / \mathrm{cm}^{-1}\right.$ \\
\hline XVIIIa & 52.12 & 160 & $\begin{array}{l}1603.10\left(\mathrm{ArC}=\mathrm{C}_{\text {str }}\right), 3010\left(=\mathrm{C}-\mathrm{H}_{\text {str }}\right), 1680.55\left(\mathrm{C}=\mathrm{O}_{\text {str }}\right) \text {, } \\
1303.01\left(\mathrm{C}=\mathrm{N}_{\mathrm{str}}\right), 997\left(\mathrm{C}-\mathrm{H}_{\text {defalkene }}\right), 1270 \text { (ether } \\
\left.\text { linkage }_{\text {str }}\right)\end{array}$ \\
\hline XVIIIlb & 55.13 & 170 & $\begin{array}{l}1680.55\left(\mathrm{C}=\mathrm{O}_{\text {str }}\right), 1303.07\left(\mathrm{C}=\mathrm{N}_{\text {str }}\right), 1603.14 \text { (Aromatic } \\
\left.\mathrm{C}=\mathrm{C}_{\text {str }}\right), 2975.93\left(=\mathrm{C}-\mathrm{H}_{\text {str }}\right) \\
1000.47\left(\mathrm{C}-\mathrm{H}_{\text {def }} \text { alkene }\right), 1270 \text { (ether linkage } \\
\text { 337t }) \\
3370.43\left(\mathrm{O}-\mathrm{H}_{\text {str }}\right) .\end{array}$ \\
\hline XVIIIc & 69.13 & 180 & $\begin{array}{l}1680.26\left(\mathrm{C}=\mathrm{O}_{\text {str }}, 1303.33\left(\mathrm{C}=\mathrm{N}_{\text {str }}\right), 1603.26 \text { (Aromatic }\right. \\
\left.\mathrm{C}=\mathrm{C}_{\text {str }}\right), 2975.96\left(=\mathrm{C}-\mathrm{H}_{\text {str }}\right), 1000.53\left(\mathrm{C}-\mathrm{H}_{\text {def }} \text { alkene }\right), \\
1252.09 \text { (ether linkage }\end{array}$ \\
\hline XVIIId & 63.18 & 120 & $\begin{array}{l}1680.17\left(\mathrm{C}=\mathrm{O}_{\text {str }}\right), 1303.08\left(\mathrm{C}=\mathrm{N}_{\text {str }}\right), 1603.04 \text { (Aromatic } \\
\left.\mathrm{C}=\mathrm{C}_{\text {str }}\right), 2974.06\left(=\mathrm{C}-\mathrm{H}_{\text {str }}\right), \\
\left.1000.35\left(\mathrm{C}-\mathrm{H}_{\text {def }} \text { alkene }\right), 1250.10 \text { (ether linkage } \text { str }\right) \\
3371.01\left(\mathrm{O}-\mathrm{H}_{\text {str }}\right)\end{array}$ \\
\hline XVIIIle & 48.19 & 200 & $\begin{array}{l}1680.01(\mathrm{C}=\mathrm{O} \text { str }), 1302.94\left(\mathrm{C}=\mathrm{N}_{\text {str }}\right), 1603.04 \text { (Aromatic } \\
\left.\mathrm{C}=\mathrm{C}_{\text {str }}\right), 2974.16\left(=\mathrm{C}-\mathrm{H}_{\text {str }}\right), 1000.57\left(\mathrm{C}-\mathrm{H}_{\text {def }}\right. \\
\text { alkene), } 1249.83(\text { ether linkage } \\
\text { str }\end{array}$ \\
\hline XVIIIf & 64.85 & 170 & $\begin{array}{l}1680.89(\mathrm{C}=\mathrm{O} \text { str) } \\
\left.\left.\mathrm{C}=\mathrm{C}_{\text {str }}\right), 2975.84 \text { ( }=\mathrm{C}-\mathrm{H}_{\text {str }}\right), 1000.38\left(\mathrm{C}=\mathrm{N}_{\text {str }}\right), 1603.19 \text { (Aromatic } \\
\text { alkene), } 1252.33 \text { (ether linkage } \mathrm{H}_{\text {str }} \text { ). }\end{array}$ \\
\hline
\end{tabular}




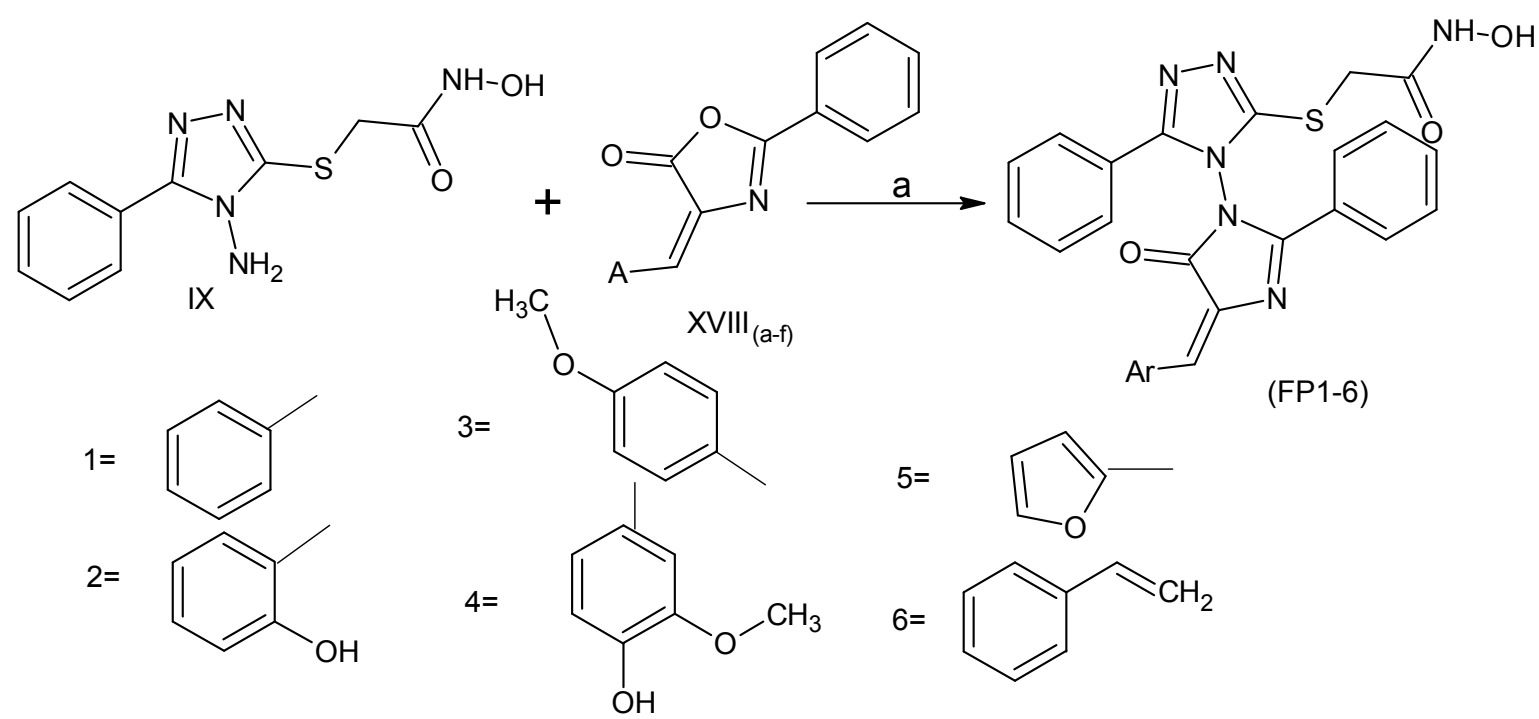

Scheme 5: (a) Pyridine, $120^{\circ} \mathrm{C}, 7$ h (FP1. 30.42\%, FP2. 28.55\%, FP3. 43.84\%, FP4. 35.21\%, FP5. 23.21\%, FP6. $25.16 \%$<smiles>[Y]C=C1N=C(c2ccccc2)N(N2C(=O)C(=C[Y7])N=C2c2ccc(OC)c(-c3nnc(SCC(=O)NO)n3-n3nc(-c4ccc(C)cc4)oc3=O)c2)C1=O</smiles>

Scheme 6: (h) Pyridine, $120^{\circ} \mathrm{C}, 7$ h (FP7. 33.12\%, FP8. 27.65\%, FP9. 33.64\%, FP10. 31.51\%, FP11. 28.18\%, FP12. 45.23\%)

\section{In vitro HDAC inhibition assay}

In vitro fluorescent histone deacetylase assay: HDAC inhibition assays were performed using the HDAC fluorescent activity assay kit. HeLa cell nuclear extract which contains a number of HDAC isozymes and other nuclear factors, was used as the source of HDAC activity. The final substrate concentration in the assay mixture was $50 \mu \mathrm{M}$. The reaction was allowed to proceed for $10 \mathrm{~min}$ at room temperature before a stop solution was added. Test compounds were prepared as $20 \mathrm{mM}$ stock solutions in DMSO and stored at $-70{ }^{\circ} \mathrm{C}$. DMSO had no significant effect on the activity of this assay at concentrations up to $5 \%$ with the final DMSO concentration in the assays of not more than $2 \%$. Assays were performed in white polystyrene 96-well half-area assay plates and measured on a Wallac 1420 fluorescent plate reader with an excitation wavelength of $355 \mathrm{~nm}$, an emission wavelength of $460 \mathrm{~nm}$, and a one sec signal averaging time [13].

\section{Evaluation of antiproliferative activity on human breast cancer cell line (MCF-7)}

Antiproliferative activity of FP1-FP12 was evaluated using Sulphorodamine B (SRB) assay method on MCF-7 cell line [14]. Cultures fixed with trichloroacetic acid were stained for $30 \mathrm{~min}$ with $0.4 \%(\mathrm{w} / \mathrm{v})$ sulforhodamine B (SRB) dissolved in $1 \%$ acetic acid. Unbound dye was removed by four washes with $1 \%$ acetic acid, and protein-bound dye was extracted with $10 \mathrm{mM}$ unbuffered Tris base [tris hydroxymethyl) aminomethane] for determination of optical density in a computer-interfaced, 96-well micro 
titer plate reader. SRB assay results were linear with the number of cells and with values for cellular protein measured by both Lowry and Bradford assays at densities ranging from sparse sub-confluence to multilayered supraconfluence. The signal-to-noise ratio at $564 \mathrm{~nm}$ was approximately 1.5 with 1,000 cells per well.

The sensitivity of SRB assay compared favorably with sensitivities of several fluorescence assays and was superior to those of both Lowry and Bradford assays and to those of 20 other visible dyes. SRB assay provides a colorimetric end point that is nondestructive, indefinitely stable, and visible to the naked eye. It provides a sensitive measure of drug-induced cytotoxicity, is useful in quantitating clonogenicity, and is well suited to high-volume, automated drug screening. SRB fluoresces strongly with laser excitation at $488 \mathrm{~nm}$ and can be measured quantitatively at the single-cell level by static fluorescence cytometry.

\section{Statistical analysis}

All the data were subjected to one-way ANOVA followed by Dunnett's test. Statistical analysis was carried out using GraphPad Prism software 5.0. Statistically significant difference was set at $p<0.05$.

\section{RESULTS}

\section{Molecular docking}

Energy minimized conformer with best Dock scores was considered for the identification of interacting amino acid residues with ligands. All the binding interactions were tabulated and diagrammatized in the (Table 1).

Table 1: Free energies of binding $(\Delta G)$ of samples FP1 - FP12 with the standard SAHA molecule

\begin{tabular}{lc}
\hline $\begin{array}{l}\text { Compound } \\
\text { code }\end{array}$ & $\begin{array}{c}\text { Free energy of } \\
\text { binding } \\
\text { (kcal/mol) }\end{array}$ \\
\hline FP1 & -7.59 \\
FP2 & -7.82 \\
FP3 & -8.64 \\
FP4 & -7.62 \\
FP5 & -7.65 \\
FP6 & -7.65 \\
FP7 & -7.64 \\
FP8 & -8.86 \\
FP9 & -7.13 \\
FP10 & -7.65 \\
FP11 & -7.63 \\
FP12 & -7.78 \\
SAHA & -7.48 \\
\hline
\end{tabular}
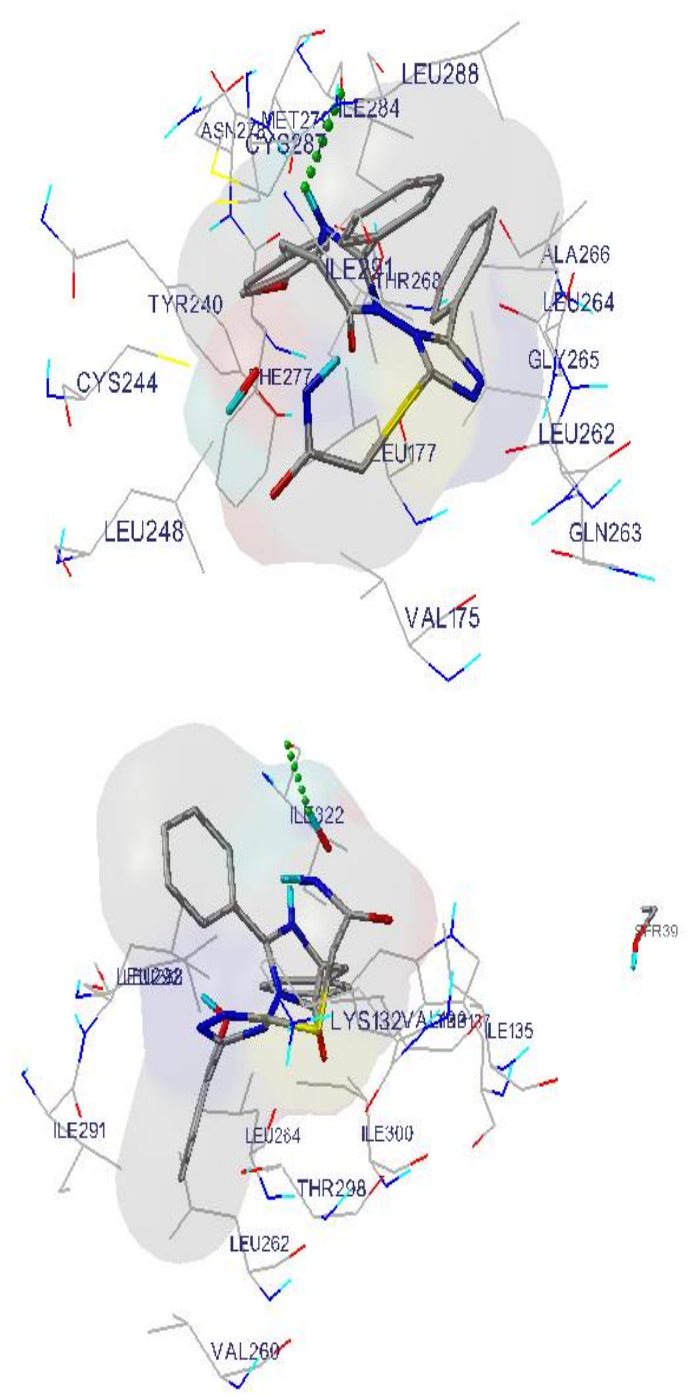

Figure 2: Molecular docking interaction of compounds FP3 and FP8 with the pdb: 1T69. Green dots represent hydrogen bond

\section{Synthesized compounds}

Twelve new hydroxyacetamide derivatives (FP1 FP12) were prepared by the condensation of different oxazolone (XVIIla-f) and appropriately substituted triazolated hydroxyacetamide (IX and XV) using reported method as per Schemes 5 and $6[11,12]$. The compounds were recrystallized using ethanol. All the compounds were characterized by detailed spectroscopic (IR, $1 \mathrm{H} \mathrm{NMR}$, Mass) analyses.

FP1: Yield $=30.42 \%$, M. $p=200^{\circ}$ C. FT-IR $(\mathrm{KBr})$ v/ $\mathrm{cm}^{-1} 769.60$ (mono substituted aromatic ring def), $1610.67\left(\mathrm{C}=\mathrm{O}_{\text {str }}\right), 1351.88\left(-\mathrm{OH}_{\text {def }}\right), 1501.17(-$ $\mathrm{NH}$ def $), 1558.15\left(\mathrm{C}=\mathrm{N}_{\text {str }}\right), 2951.81\left(\mathrm{C}=\mathrm{S}_{\text {str }}\right)$, $1306.38\left(\mathrm{~N}-\mathrm{N}=\mathrm{C}_{\text {str }}\right.$ ) 1573.58 (nitrogen containing heterocyclic ring in combination of $\mathrm{C}=\mathrm{C}) .{ }^{1} \mathrm{H}$ NMR DMSO (300 MHz) $\delta$ ppm: 7.31-8.16 (m, $15 \mathrm{H}, \mathrm{Ar}-\mathrm{H}), 7.86(\mathrm{~s}, 1 \mathrm{H}, \mathrm{NH}), 3.33$ (1H aliphatic $\mathrm{CH}_{2}$ ), $\delta 2.50$ (1Hwas observed for hydroxamic 
$\mathrm{OH}$ proton). $\quad \mathbf{C}_{26} \mathbf{H}_{20} \mathbf{N}_{6} \mathbf{O}_{3} \mathbf{S}$ : calculated C: $62.90 \%, \quad \mathrm{H}: \quad 4.03 \%, \quad \mathrm{~N}: 16.9 \% . \quad \mathbf{C}_{26} \mathrm{H}_{20} \mathbf{N}_{6} \mathbf{O}_{3} \mathrm{~S}:$ found: C: $61.30 \%, \mathrm{H}: 4.02 \%, \mathrm{~N}: 16.5 \%$. $(\mathrm{M}+1)$ 496.12 (EIMS).

FP2: Yield $=28.55 \%, M . p=210^{\circ} \mathrm{C} . \mathrm{FT}-\mathrm{IR}(\mathrm{KBr}) \mathrm{v} /$ $\mathrm{cm}^{-1} 769.70$ (mono substituted aromatic ring def), $1610.51(\mathrm{C}=\mathrm{O}$ str $), 1351.64 \mathrm{C}=\mathrm{N}$ str as well as $\left.\mathrm{OH}{ }_{\text {def }}\right), 1500.50\left(-\mathrm{NH}{ }_{\text {def }}\right), 2952.52$ (C=S str), $1306.06\left(\mathrm{~N}-\mathrm{N}=\mathrm{C}_{\text {str }}\right)$ 1573.21 (nitrogen containing heterocyclic ring in combination of $\mathrm{C}=\mathrm{C}_{\text {str }}$ ), $1558.03\left(\mathrm{C}=\mathrm{N}_{\text {str }}\right) .{ }^{1} \mathrm{H}$ NMR DMSO $(300 \mathrm{MHz}) \delta$ ppm: $\delta 7.87(\mathrm{~s}, 1 \mathrm{H}, \mathrm{N}-\mathrm{H}), \delta 7.61(\mathrm{~m}, \mathrm{Ar}-\mathrm{H}), \delta$ $3.32\left(1 \mathrm{H}\right.$ aliphatic $\left.\mathrm{CH}_{2}\right), \delta 2.50(\mathrm{~s}, 1 \mathrm{H}, \mathrm{OH})$. $\mathrm{C}_{26} \mathrm{H}_{20} \mathrm{~N}_{6} \mathrm{O}_{4} \mathrm{~S}$ : calculated C: $60.90 \%, \mathrm{H}: 3.92 \%$, $\mathrm{N}: 16.47 \% . \mathbf{C}_{26} \mathbf{H}_{20} \mathbf{N}_{6} \mathbf{O}_{4}$ S: found: C: $60.14 \%, \mathrm{H}$ : $3.89 \%, \mathrm{~N}: 16.4 \%$. (M+1) 510.32 (EIMS).

FP3: Yield $=43.84 \%, M . p=158^{\circ} \mathrm{C}$. FT-IR $(\mathrm{KBr}) \mathrm{v} /$ $\mathrm{cm}^{-1} 843.26$ (para substituted aromatic ring def), $1610.42(\mathrm{C}=\mathrm{O}$ str $), 1351.56(\mathrm{C}=\mathrm{N}$ str), $1501.43(-$ $\mathrm{NH}$ def $), 2928.51\left(\mathrm{C}=\mathrm{S}_{s t r}\right), 1295.23\left(\mathrm{~N}-\mathrm{N}=\mathrm{C}_{s t r}\right)$, $1558.03(\mathrm{C}=\mathrm{N}$ str $), 1265.28\left(\mathrm{Ar}-\mathrm{OCH}_{3}\right), 3102(\mathrm{NH}$ $s t r), 3280.51\left(\mathrm{OH}_{s t r}\right) .{ }^{1} \mathrm{H}$ NMR DMSO $(300 \mathrm{MHz}) \delta$ ppm: $\delta 7.87(\mathrm{~s}, 1 \mathrm{H}, \mathrm{N}-\mathrm{H}), \delta 7.61(\mathrm{~m}, 14 \mathrm{H}, \mathrm{Ar}-\mathrm{H})$, $\delta 3.38\left(\mathrm{~d}, 2 \mathrm{H}\right.$ aliphatic $\left.\mathrm{CH}_{2}\right), \delta 2.50(\mathrm{~s}, 1 \mathrm{H}, \mathrm{OH})$, $3.93\left(\mathrm{t}, 3 \mathrm{H}, \mathrm{OCH}_{3}\right.$ at $4^{\text {th }}$ position of aromatic ring). $\mathrm{C}_{27} \mathrm{H}_{22} \mathrm{~N}_{6} \mathrm{O}{ }_{4} \mathrm{~S}$ : calculated C: $61.53 \%, \mathrm{H}: 4.18 \%$, $\mathrm{N}: 15.96 \%$. $\mathbf{C}_{27} \mathbf{H}_{22} \mathbf{N}_{6} \mathbf{O}_{4}$ S: found $\mathrm{C}: 60.50 \%, \mathrm{H}$ : $4.10 \%, \mathrm{~N}: 15.50 \%$. (M+1) 526.32 (EIMS) and 179.0266 the main fragmented portion.

FP4: Yield $=35.21 \%, M . p=155^{\circ} \mathrm{C} . \mathrm{FT}-\mathrm{IR}(\mathrm{KBr}) \mathrm{v} /$ $\mathrm{cm}^{-1} 802.00$ (para substituted aromatic ring def), 1609.38 ( $\mathrm{C}=\mathrm{O}$ str) $), 1351.56$ ( $\mathrm{C}=\mathrm{N}$ str), $1501.43(-$ $\left.\mathrm{NH}{ }_{d e f}\right), 2927.45(\mathrm{C}=\mathrm{S}$ str $), 1308.14\left(\mathrm{~N}-\mathrm{N}=\mathrm{C}_{s t r}\right)$, 1573.91(nitrogen containing heterocyclic ring in combination of $\mathrm{C}=\mathrm{C}), 1558.03(\mathrm{C}=\mathrm{N}$ str $), 1266$ $\left(\mathrm{Ar}-\mathrm{OCH}_{3}\right) .{ }^{1} \mathrm{H}$ NMR DMSO $(300 \mathrm{MHz}) \delta \mathrm{ppm}: \delta$ $7.86(\mathrm{~s}, 1 \mathrm{H}, \mathrm{N}-\mathrm{H}), \delta 7.45(\mathrm{~m}, 14 \mathrm{H}, \mathrm{Ar}-\mathrm{H}), \delta 3.38$ (d, 2H aliphatic $\mathrm{CH}_{2}$ ), $\delta 2.50(\mathrm{~s}, 1 \mathrm{H}, \mathrm{OH}), 3.91$ (t, $3 \mathrm{H}, \mathrm{OCH}_{3}$ at the $\mathrm{C}-3$ position of aromatic ring), $8.82\left(\mathrm{~s}, \quad 1 \mathrm{H}, \quad\right.$ Phenolic OH). $\quad \mathbf{C}_{27} \mathbf{H}_{22} \mathbf{N}_{6} \mathbf{O}_{5} \mathbf{S}$ : calculated C: $59.71 \%, \mathrm{H}: 3.93 \%, \mathrm{~N}: 15.02 \%$. $\mathrm{C}_{27} \mathrm{H}_{22} \mathrm{~N}_{6} \mathrm{O}_{5} \mathrm{~S}$ : found C: $59.574 \%, \mathrm{H}: 3.85 \%, \mathrm{~N}$ : 14.88\%. (M+1) 559.0299 (EIMS) and the main fragmented portion is 179.0266 .

FP5: Yield $=23.21 \%, M . p=205^{\circ} \mathrm{C} . \mathrm{FT}-\mathrm{IR}(\mathrm{KBr}) \mathrm{v} /$ $\mathrm{cm}^{-1} 790.37$ (mono substituted aromatic ring def), 1601.61 ( $\mathrm{C}=\mathrm{O}$ str), 1340.57 ( $\mathrm{C}=\mathrm{N}$ str $), 1308.12$ $\left(\mathrm{N}-\mathrm{N}=\mathrm{C}\right.$ str), 1266.29 (etheric linkage). ${ }^{1} \mathrm{H}$ NMR DMSO $(300 \mathrm{MHz}) \delta \mathrm{ppm}: \delta 7.85(\mathrm{~s}, 1 \mathrm{H}, \mathrm{N}-\mathrm{H}), \delta$ $7.57(\mathrm{~m}, 10 \mathrm{H}, \mathrm{Ar}-\mathrm{H}), \delta 3.35$ (d, $2 \mathrm{H}$ aliphatic $\mathrm{CH}_{2}$ ), $\delta 2.50(\mathrm{~s}, 1 \mathrm{H}, \mathrm{OH}), 7.64(\mathrm{~s}, 1 \mathrm{H}$, furan $\mathrm{H})$. $\mathrm{C}_{24} \mathrm{H}_{18} \mathrm{~N}_{6} \mathrm{O}_{4} \mathrm{~S}$ : calculated C: $59.19 \%$, H: $3.70 \%$, $\mathrm{N}: 17.28 \% . \mathrm{C}_{24} \mathrm{H}_{18} \mathrm{~N}_{6} \mathrm{O}_{4} \mathrm{~S}$ : found C: $58.35 \%, \mathrm{H}$ : $3.63 \%, \mathrm{~N}: 16.96 \%$. (M+1) 486.02 (EIMS) and 179.0268 .
FP6: Yield $=25.96 \%$, M.p $=188^{\circ} \mathrm{C} . \mathrm{FT}-\mathrm{IR}(\mathrm{KBr}) \mathrm{v} /$ $\mathrm{cm}^{-1} 790.374$ (mono substituted aromatic ring def), $1609.98 \quad(\mathrm{C}=\mathrm{O} \quad s t r), 1351.19 \quad(\mathrm{C}=\mathrm{N} \quad s t r)$, $1307.49\left(\mathrm{~N}-\mathrm{N}=\mathrm{C}_{s t r}\right), 1266.29$ (etheric linkage). ${ }^{1} \mathrm{H}$ NMR DMSO (300 MHz) $\delta$ ppm: $\delta 7.86(\mathrm{~s}, 1 \mathrm{H}, \mathrm{N}-$ $\mathrm{H}), \delta 7.57(\mathrm{~m}, 10 \mathrm{H}, \mathrm{Ar}-\mathrm{H}), \delta 3.32$ (d, 2H aliphatic $\left.\mathrm{CH}_{2}\right), \delta 2.50(\mathrm{~s}, 1 \mathrm{H}, \mathrm{OH}), 3.44\left(\mathrm{~s}, 1 \mathrm{H}, \mathrm{CH}_{3}\right)$. $\mathrm{C}_{2} 1 \mathrm{H}_{18} \mathrm{~N}_{6} \mathrm{O}_{3} \mathrm{~S}$ : calculated C: $58.00 \%, \mathrm{H}: 4.15 \%$, $\mathrm{N}: 19.39 \%$. $\mathbf{C}_{2} \mathbf{1} \mathbf{H}_{18} \mathbf{N}_{6} \mathbf{O}_{3}$ S: found C: $57.613 \%, \mathrm{H}$ : 4.02\%, N: 18.95\%. (M+1) 433.2019 (EIMS).

FP7: Yield $=33.12 \%$, M. $p=190^{\circ} \mathrm{C} . \mathrm{FT}-\mathrm{IR}(\mathrm{KBr}) \mathrm{v} /$ $\mathrm{cm}^{-1} 749.59$ (mono substituted aromatic ring def), $1592.68\left(\mathrm{C}=\mathrm{O}{ }_{s t r}\right), 1384.68 \mathrm{C}=\mathrm{N}$ str as well as $\mathrm{OH}{ }_{\text {def }}$ ), 1487.21 ( $-\mathrm{NH}{ }_{\text {def }}$ ), 2932.62 ( $\mathrm{C}=\mathrm{S}{ }_{\text {str }}$ ), $1291.72\left(\mathrm{~N}-\mathrm{N}=\mathrm{C}_{\text {str }}\right) .{ }^{1} \mathrm{H}$ NMR DMSO $(300 \mathrm{MHz})$ $\delta$ ppm: $\delta 7.84(\mathrm{~s}, 1 \mathrm{H}, \mathrm{N}-\mathrm{H}), \delta 7.23(\mathrm{~m}, \mathrm{Ar}-\mathrm{H}), \delta$ $3.91\left(1 \mathrm{H}\right.$ aliphatic $\left.\mathrm{CH}_{2}\right), \delta 2.72(\mathrm{~d}, 1 \mathrm{H}, \mathrm{Ar}-\mathrm{OH}) \delta$ $2.50(\mathrm{~s}, 1 \mathrm{H}, \mathrm{OH}), \mathrm{C}_{26} \mathrm{H}_{20} \mathrm{~N}_{6} \mathrm{O}_{4} \mathrm{~S}$ : calculated C: $60.94 \%, \quad \mathrm{H}: 3.91 \%, \mathrm{~N}: 16.40 \% . \quad \mathbf{C}_{26} \mathbf{H}_{20} \mathbf{N}_{6} \mathbf{O}_{4} \mathbf{S}:$ found C: $60.13 \%, \mathrm{H}: 3.85 \%, \mathrm{~N}: 15.85 \%$. $(\mathrm{M}+1)$ 512.58 (EIMS).

FP8: Yield $=27.65 \%, M . p=205^{\circ} \mathrm{C}$. FT-IR $(\mathrm{KBr}) \mathrm{v} /$ $\mathrm{cm}^{-1} 750.87$ (mono substituted aromatic ring def), $1592.37(\mathrm{C}=\mathrm{O}$ str $), 1384.68(\mathrm{C}=\mathrm{N}$ str as well as $\left.\mathrm{OH}{ }_{\text {def }}\right), 1487.40$ ( $-\mathrm{NH}$ def $), 2932.21$ ( $\mathrm{C}=\mathrm{S}$ str $)$, $1291.85\left(\mathrm{~N}-\mathrm{N}=\mathrm{C}_{s t r}\right) .{ }^{1} \mathrm{H}$ NMR DMSO $(300 \mathrm{MHz})$ $\delta$ ppm: $\delta 7.81(\mathrm{~s}, 1 \mathrm{H}, \mathrm{N}-\mathrm{H}), \delta 7.29(\mathrm{~m}, \mathrm{Ar}-\mathrm{H}), \delta$ $3.91\left(1 \mathrm{H}\right.$ aliphatic $\left.\mathrm{CH}_{2}\right), \delta 2.50(\mathrm{~d}, 1 \mathrm{H}, \mathrm{Ar}-\mathrm{OH}) \delta$ $2.50(\mathrm{~s}, 1 \mathrm{H}, \mathrm{OH}) . \mathrm{C}_{26} \mathrm{H}_{20} \mathrm{~N}_{6} \mathrm{O}_{5} \mathrm{~S}$ : calculated C: $59.21 \%, \quad \mathrm{H}: 3.78 \%, \quad \mathrm{~N}: 15.90 \% . \quad \mathbf{C}_{26} \mathrm{H}_{20} \mathbf{N}_{6} \mathrm{O}_{5} \mathrm{~S}:$ found C: $60.143 \%, \mathrm{H}: 3.65 \%, \mathrm{~N}: 15.58 \%$. $(\mathrm{M}+1)$ 528.52 (EIMS).

FP9: Yield $=33.64 \%, \mathrm{M} . \mathrm{p}=185^{\circ} \mathrm{C}$. FT-IR $(\mathrm{KBr}) \mathrm{v} /$ $\mathrm{cm}^{-1} 763.44$ (mono substituted aromatic ring def), $1593.84(\mathrm{C}=\mathrm{O}$ str $), 1384.65(\mathrm{C}=\mathrm{N}$ str as well as $\mathrm{OH}$ def), 1512.01 ( $-\mathrm{NH}{ }_{\text {def }}$ ), 2933.89 ( $\mathrm{C}=\mathrm{S}_{\mathrm{str}}$ ), $1321.24\left(\mathrm{~N}-\mathrm{N}=\mathrm{C}_{\mathrm{str}}\right) .{ }^{1} \mathrm{H}$ NMR DMSO $(300 \mathrm{MHz})$ $\delta$ ppm: $\delta 7.79(\mathrm{~s}, 1 \mathrm{H}, \mathrm{N}-\mathrm{H}), \delta 7.39(\mathrm{~m}, \mathrm{Ar}-\mathrm{H}), \delta$ $3.81\left(1 \mathrm{H}\right.$ aliphatic $\left.\mathrm{CH}_{2}\right), \delta 2.66(\mathrm{~d}, 1 \mathrm{H}, \mathrm{Ar}-\mathrm{OH}) \delta$ $2.50(\mathrm{~s}, 1 \mathrm{H}, \mathrm{OH}) . \mathrm{C}_{27} \mathrm{H}_{20} \mathrm{~N}_{6} \mathrm{O}_{5} \mathrm{~S}$ : calculated $\mathrm{C}$ : $59.77 \%, \quad \mathrm{H}: 3.69 \%, \quad \mathrm{~N}: 15.49 \% . \quad \mathbf{C}_{27} \mathrm{H}_{20} \mathbf{N}_{6} \mathrm{O}_{5} \mathrm{~S}:$ found C: $59.90 \%, \mathrm{H}: 3.52 \%, \mathrm{~N}: 15.04 \%$. $(\mathrm{M}+1)$ 542.54 (EIMS).

FP10: Yield $=31.51 \%, M . p=192^{\circ} \mathrm{C} . \mathrm{FT}-\mathrm{IR}(\mathrm{KBr})$ $\mathrm{V} / \mathrm{cm}^{-1} 807.57$ (para substituted aromatic ring def), $1644.98 \quad(\mathrm{C}=\mathrm{O} \quad$ str $), 1384.71 \quad(\mathrm{C}=\mathrm{N}$ str $)$, $1501.04\left(-\mathrm{NH}_{\text {def }}\right), 2932.40\left(\mathrm{C}=\mathrm{S}_{\text {str }}\right), 1332.97(\mathrm{~N}-$ $\mathrm{N}=\mathrm{C} \quad$ str),$\quad 1594.13 \quad$ (nitrogen containing heterocyclic ring in combination of $\mathrm{C}=\mathrm{C}$ ), $1558.03\left(\mathrm{C}=\mathrm{N}_{\text {str }}\right), 1157.10\left(\mathrm{Ar}-\mathrm{OCH}_{3}\right) .{ }^{1} \mathrm{H}$ NMR DMSO (300 MHz) $\delta$ ppm: $\delta 7.86(\mathrm{~s}, 1 \mathrm{H}, \mathrm{N}-\mathrm{H}), \delta$ $7.14(\mathrm{~m}, 14 \mathrm{H}, \mathrm{Ar}-\mathrm{H}), \delta 3.31$ (d, $2 \mathrm{H}$ aliphatic $\mathrm{CH}_{2}$ ), $\delta 2.50(\mathrm{~s}, 1 \mathrm{H}, \mathrm{OH}), 3.31\left(\mathrm{t}, 3 \mathrm{H}, \mathrm{OCH}_{3}\right.$ at $3^{\text {rd }}$ position of aromatic ring), $\delta 2.50(\mathrm{~s}, 1 \mathrm{H}, \mathrm{OH})$. $\mathrm{C}_{27} \mathrm{H}_{22} \mathrm{~N}_{6} \mathrm{O}_{6} \mathrm{~S}$ : calculated C: $57.96 \%, \mathrm{H}: 3.94 \%$ 
$\mathrm{N}: 15.05 \% . \mathbf{C}_{27} \mathbf{H}_{22} \mathbf{N}_{6} \mathbf{O}_{6} \mathbf{S}$ : found C: $58.13 \%, \mathrm{H}$ $4.02 \%, \mathrm{~N}: 14.85 \%$. (M+1) 558.84 (EIMS).

FP11: Yield $=28.18 \%$, M. $p=212^{\circ}$ C. FT-IR $(\mathrm{KBr})$ $\mathrm{V} / \mathrm{cm}^{-1} 1807.41$ (mono substituted aromatic ring def $), \quad 1644.54 \quad(\mathrm{C}=\mathrm{O}$ str $), 1329.34 \quad(\mathrm{C}=\mathrm{N}$ str $)$, $1308.12\left(\mathrm{~N}-\mathrm{N}=\mathrm{C}_{\text {str }}\right), 1266.29$ (etheric linkage). ${ }^{1} \mathrm{H}$ NMR DMSO $(300 \mathrm{MHz}) \delta \mathrm{ppm}: \delta 7.58(\mathrm{~s}, 1 \mathrm{H}, \mathrm{N}-$ $\mathrm{H}), \delta 7.12(\mathrm{~m}, 10 \mathrm{H}, \mathrm{Ar}-\mathrm{H}), \delta 3.31(\mathrm{~d}, 2 \mathrm{H}$ aliphatic $\mathrm{CH}_{2}$ ), $\delta 2.50$ (s, 1H, OH), 8.11 (s, 1H, furan $\mathrm{H}$ ). $\mathbf{C}_{24} \mathbf{H}_{18} \mathbf{N}_{6} \mathbf{O}_{5} \mathbf{S}$ : calculated C: $57.31 \%, \mathrm{H}: 3.58 \%$, $\mathrm{N}: 16.73 \% . \mathbf{C}_{24} \mathbf{H}_{18} \mathbf{N}_{6} \mathbf{O}_{5}$ S: found C: $57.30 \%, \mathrm{H}$ : $3.45 \%$, N: 15.98\%. (M+1) 502.90 (EIMS).

FP12: Yield $=45.23 \%, M . p=188^{\circ} \mathrm{C} . \quad F T-I R(\mathrm{KBr})$ $\mathrm{v} / \mathrm{cm}^{-1} 807.48$ (mono substituted aromatic ring def), $1644.98 \quad(\mathrm{C}=\mathrm{O}$ str $), 1331.87 \quad(\mathrm{C}=\mathrm{N}$ str $)$, 1308.76 ( $\left.\mathrm{N}-\mathrm{N}=\mathrm{C}_{\text {str }}\right), 1266.65$ (etheric linkage). ${ }^{1} \mathrm{H}$ NMR DMSO (300 MHz) $\delta$ ppm: $\delta 7.859$ (s, $1 \mathrm{H}, \mathrm{N}-\mathrm{H}), \delta 7.138(\mathrm{~m}, 15 \mathrm{H}, \mathrm{Ar}-\mathrm{H}), \delta 3.311(\mathrm{~d}, 2 \mathrm{H}$ aliphatic $\left.\mathrm{CH}_{2}\right), \delta 2.500(\mathrm{~s}, 1 \mathrm{H}, \mathrm{OH}), \delta 7.480$, $5.265(\mathrm{~d}, \quad 1 \mathrm{H}$, ethylene group of cinnamaldehyde). $\mathbf{C}_{28} \mathbf{H}_{22} \mathbf{N}_{6} \mathbf{O}_{4} \mathbf{S}$ : calculated $\mathrm{C}$ : $62.39 \%, \mathrm{H}: 4.08 \%, \mathrm{~N}: 15.61 \%, \mathbf{C}_{28} \mathbf{H}_{22} \mathbf{N}_{6} \mathbf{O}_{4} \mathbf{S}:$ found $\mathrm{C}: 62.85 \%, \mathrm{H}: 3.92 \%, \mathrm{~N}: 14.85 \% .(\mathrm{M}+1)$ 538.65 (EIMS).

\section{In vitro HDAC inhibition}

The in vitro HDAC inhibition activities of compounds FP1 - 12 are reported in terms of \% inhibitory concentration $\left(\mathrm{IC}_{50}\right)$. All the results were presented in Table 3 and Figure 4.<smiles>[R2]C=C1N=C(c2ccccc2)N(n2c(SCC(=O)NO)nnc2-c2ccccc2[R1])C1=O</smiles>

Figure 3: Basic pharmacophore of the synthesized structure

\section{Antiproliferative activity against MCF-7 cell line}

The in vitro cytotoxic activity of compounds FP1 12 are reported in terms of $\% \mathrm{GI}_{50}$ and the data are shown in Table 4.

Table 3: In vitro HDAC inhibition activity of FP1 - FP12

\begin{tabular}{lccc}
\hline Compound code & R1 & R2 & IC $_{50}(\boldsymbol{\mu M})$ \\
\hline FP1 & $\mathrm{H}$ & Phenyl & 6.2 \\
FP2 & $\mathrm{H}$ & 2-Hydroxyphenyl & 5.4 \\
FP3 & $\mathrm{H}$ & 4-Mehoxyphenyl & 2.1 \\
FP4 & $\mathrm{H}$ & 4-Hydroxy-3-Methoxyphenyl & 0.16 \\
FP5 & $\mathrm{H}$ & Furfuryl & 5.8 \\
FP6 & $\mathrm{H}$ & 1-Ethenylbenzene & 5.3 \\
FP7 & $\mathrm{OH}$ & Phenyl & 5.2 \\
FP8 & $\mathrm{OH}$ & 2-Hydroxyphenyl & 4.5 \\
FP9 & $\mathrm{OH}$ & 4-Methoxyphenyl & 1.6 \\
FP10 & $\mathrm{OH}$ & 4-Hydroxy-3-Methoxyphenyl & 0.09 \\
FP11 & $\mathrm{OH}$ & Furfuryl & 4.3 \\
FP12 & $\mathrm{OH}$ & 1-Ethenylbenzene & 4.9 \\
SAHA & --- & ---------- & 0.057 \\
\hline
\end{tabular}

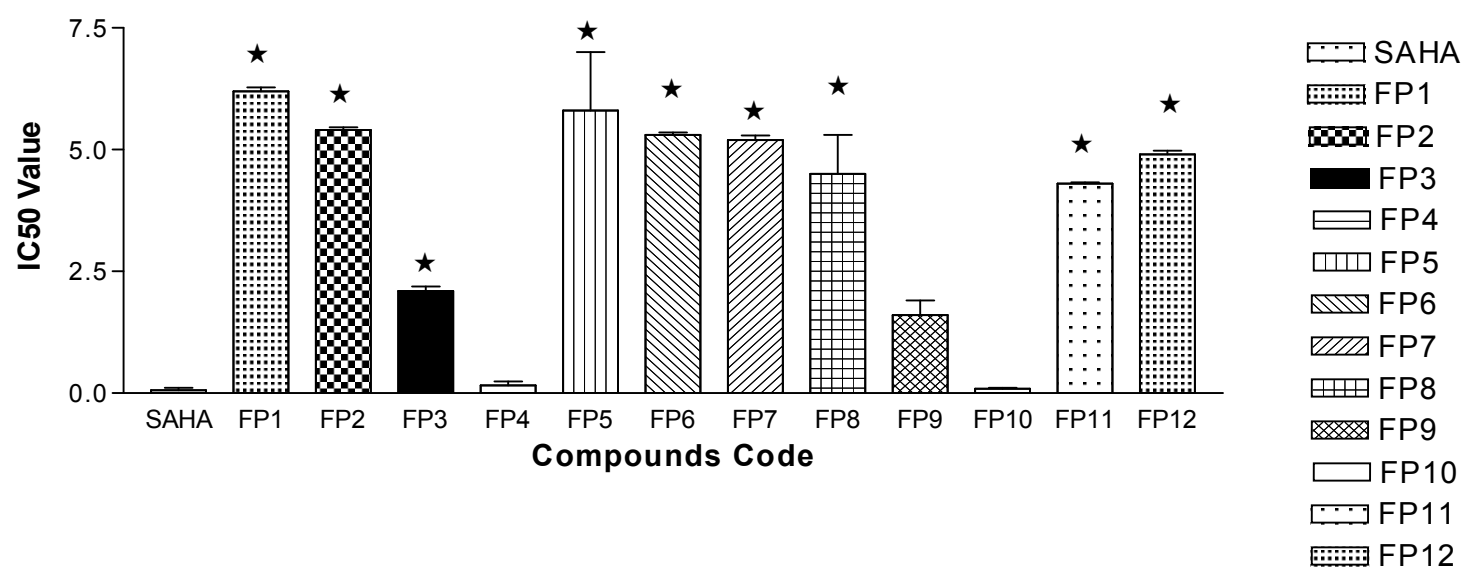

Figure 4: In vitro HDAC Inhibitory Activity against HDAC enzyme mixture derived HeLa cervical carcinoma cells of Sample FP1-FP12 with Standard SAHA 
Table 4: In vitro cytotoxic profile of FP1 - FP12 against MCF-7

\begin{tabular}{|c|c|c|c|}
\hline \multirow[t]{2}{*}{ Entry } & \multicolumn{2}{|c|}{ Anti MCF-7 Cell Line Activity ${ }^{a}$} & \\
\hline & $\mathbf{L C}_{50}$ & $\mathrm{TGI}^{\mathrm{b}}$ & $\mathbf{G I}_{50} \mathbf{b}^{\mathrm{b}}$ \\
\hline FP1 & $>80$ & $>80$ & 57.3 \\
\hline FP2 & $>80$ & $>80$ & 57.8 \\
\hline FP3 & $>80$ & $>80$ & 54.2 \\
\hline FP4 & $>80$ & $>80$ & 46.8 \\
\hline FP5 & $>80$ & $>80$ & 53.2 \\
\hline FP6 & $>80$ & $>80$ & 52.2 \\
\hline FP7 & $>80$ & $>80$ & 46.3 \\
\hline FP8 & $>80$ & $>80$ & 41.0 \\
\hline FP9 & $>80$ & $>80$ & 22.8 \\
\hline FP10 & $>80$ & $>80$ & 24.9 \\
\hline FP11 & $>80$ & $>80$ & 55.3 \\
\hline FP12 & $>80$ & $>80$ & 49.2 \\
\hline $\mathrm{ADR}^{\mathrm{c}}$ & 58.8 & 18.6 & -50.2 \\
\hline
\end{tabular}

${ }^{a}$ Each value is the mean of three independent experiments; ${ }^{b} \mathrm{LC}_{50}=$ concentration of drug causing $50 \%$ cell kill; $\mathrm{TGI}=$ concentration of drug causing total inhibition of cell growth; $\mathrm{GI}_{50}=$ concentration of drug causing $50 \%$ inhibition of cell growth; ${ }^{\mathrm{C}} \mathrm{ADR}=$ adriamycin (positive control)

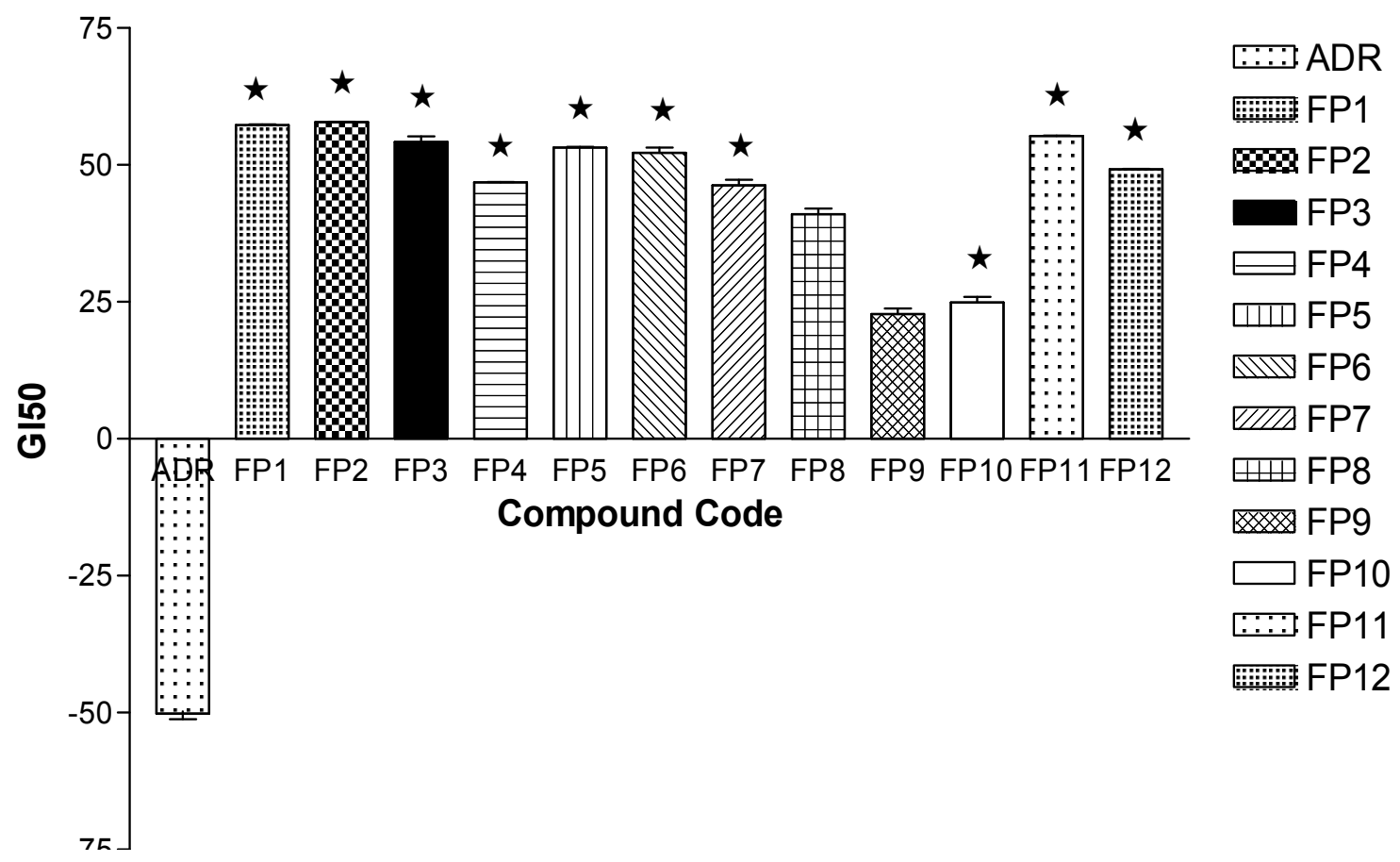

Fig 4: In vitro Growth Inhibition on MCF-7 by Sample FP1-FP12 with standard adriamycin

\section{DISCUSSION}

Docking result suggested that compound FP3, FP8 have shown a significant favorable free energy of binding (-) $8.64 \mathrm{kcal} / \mathrm{mol}$ and (-) 8.86 $\mathrm{Kcal} / \mathrm{mol}$ which seems to be much close to that of the reference standard SAHA with -7.48 $\mathrm{kcal} / \mathrm{mol}$. In the Fig 2 showed that the surrounding residues of FP3 the surrounded residues were VAL 175, LEU 262, GLY 265, LEU 264, TYR 240, ILE 284, THR 268 and in the case of FP8 the surrounding residues were ALA 38, LEU 31, TRP 14, PHE 152, LYS 33, SER 39, ARG 37 which was subsequently present in the receptor voxel. HDAC8 receptor is a metal activated enzyme which consists of $\mathrm{Zn} 378$ residue in its active site and in all the cases the docked molecules are present within a suitable range. All the synthesized molecules are characterized by FTIR, ${ }^{1} \mathrm{H}$ NMR, elemental Analysis, and mass Spectrophotometer. As per the spectral data of compound FP1-FP6, FTIR and ${ }^{1} \mathrm{H}$ NMR showed presence of characteristic peak around $1600 \mathrm{~cm}^{-1}, 3100 \mathrm{~cm}^{-1}, 3300 \mathrm{~cm}^{-1}$ due to $\mathrm{C}=\mathrm{O},-\mathrm{OH},-\mathrm{NH}$ stretching, around 3000 $\mathrm{cm}^{-1}$ for phenyl group, $1380 \mathrm{~cm}^{-1}$ for the etheric linkage and $\delta 2.500 \mathrm{ppm}$, around $7.200 \mathrm{ppm}$, around $7.800 \mathrm{ppm}$, around $3.38 \mathrm{ppm}$ due to hydroxamic $\mathrm{OH}$, Aromatic $-\mathrm{H}$, $-\mathrm{NH},-\mathrm{CH}_{2}$ group respectively but the presence of para amino 
group of the triazole moiety $\delta 5.793 \mathrm{ppm}$ is missing the final set of compound. In the case of spectral data of compound FP7-FP12, as per the ${ }^{1} \mathrm{H}$ NMR absences of para amino group of the triazole moiety $\delta 5.622$ ppm. Further, the structures were established by mass spectra data accordance to their molecular formula. The activity of new compounds FP1 - FP12 against HDAC enzyme mixture derived HeLa cervical carcinoma cells reported in Table 3 suggest that compounds (FP10, FP4, FP9) were showed apparently higher $\mathrm{IC}_{50}$ value as $0.09 \mu \mathrm{M}, 0.16$ $\mu \mathrm{M}, 1.6 \mu \mathrm{M}, 22.8 \mu \mathrm{M}$ and $24.9 \mu \mathrm{M}$ respectively as comparison to the standard SAHA molecule with $0.057 \mu \mathrm{M}$, among the result only FP10, FP9, FP4 were non-significantly varied from standard molecule data. However in the case of activity of new compounds FP1 - FP12 against MCF-7 cell line reported in Table 4 only FP9 showed relative $\mathrm{GI}_{50}$ Value with $22.8 \mu \mathrm{M}$ as comparison to control Adriamycin (ADR) with $\mathrm{GI}_{50}$ value - 50.2 $\mu \mathrm{M}$, among the result only FP9 was nonsignificantly varied from standard molecule data.

\section{CONCLUSION}

Hydroxyacetamide derivatives have been successfully synthesized and characterized by FTIR, NMR, elemental analysis and mass spectrometry. They exhibited inhibitory activity against HDAC enzyme mixture-derived HeLa cervical carcinoma cells and MCF-7 cell line. Further investigations are required the full activity profile and toxicological properties of the derivatives.

\section{DECLARATIONS}

\section{Acknowledgement}

The authors are grateful to Central Instrumental Facility, CDRI, Lucknow; Dr Arti Juvekar, Principal, Department of Pharmacology and Toxicology, ACTREC; Tata Cancer Research Institute, Navi Mumbai and Dr Verma Ram for their Support.

\section{Conflict of Interest}

No conflict of interest associated with this work.

\section{Contribution of Authors}

The authors declare that this work was done by the authors named in this article and all liabilities pertaining to claims relating to the content of this article will be borne by them.

\section{REFERENCES}

1. Ragno R, Mai A, Massa S, Cerbara I, Bottoni $P$, Scatena $R$, Jesacher F, Loidl P, Gerald B. 3-(4-Aroyl-1-methyl1H-pyrrol-2-yl)-N-hydroxy-2-propenamides as a New Class of Synthetic Histone Deacetylase Inhibitors. Discovery of Novel Lead Compounds through StructureBased Drug Design and Docking Studies. J Med Chem 2004; 47: 1351-1359.

2. Davis A, Hulme KL, Wilson GT, Mccord TJ. In vitro antimicrobial activity of some cyclic hydroxamic acids and related lactams. Antimicro agents and chemothera 1978; 13: 542- 544.

3. Kim DK, Lee JY, Kim JS, Ryu JH, Choi JY, JW Lee, Im GJ, Kim TKJ. Synthesis and biological evaluation of 3(4-substituted-phenyl)-N-hydroxy-2-propenamides, a new class of histone deacetylase inhibitors. J Med Chem 2003; 46: 5745-5751.

4. $L u Q$, Wang DS, Chen CS, Hu YD. Structure-Based Optimization of Phenylbutyrate-Derived Histone Deacetylase Inhibitors. J Med Chem 2005; 48: 55305535.

5. Belvedere S, Witter DJ, Yan J, Secrist JP, Richon V, Miller TA. Aminosuberoyl Hydroxamic Acids (Ashas): A Potent New Class of HDAC Inhibitors. Bioorg Med Chem Lett 2007; 17: 3969-3971.

6. Mai A, Massa S, Lavu S, Simeoni S, Ragno R. Design, Synthesis, And Biological Evaluation of Sirtinol Analogues As Class lii Histone/Protein Deacetylase (Sirtuin) Inhibitors. J Med Chem 2005; 48: 7789-7795.

7. Goodsell DS, Morris GM, Olson AJ. Automated Docking of Flexible Ligands: Applications of Autodock. J Mol Recognit 1996; 9: 1-5.

8. Morris GM, Goodsell DS, Halliday RS, Huey R, William E, Hart WE, Belew RK, Olson AJ. Automated docking using a Lamarckian genetic algorithm and an empirical binding free energy function. J Comput Chem 1998; 19: 1639.

9. Sousa SF, Fernandes PA, Ramos MJ. Protein-Ligand Docking: Current Status and Future Challenges. PROTEINS 2006; 65: 15.

10. Huey R, Morris GM, Olson AJR, Goodsell DS. A Semiempirical Free Energy Force Field with ChargeBased Desolvation. J Comput Chem 2007; 28: 1145.

11. Reid JR, Heindel NDJ. Improved syntheses of 5substituted-4-amino-3-mercapto-(4H)-1,2,4-triazoles. Heterocyclic Chem 1976; 13: 925.

12. Siddiqui SA, Bhusare SR, Jarikote DV, Pawar RP, Vibhute YB. New Novel synthesis and antibacterial activity of 1-(substituted phenyl)-2-phenyl-4-(3'-halo, 4'hydroxy, 5'-methoxy benzylidene)-imidazole-5-ones. Bull Kor Chem Soc 2001; 22: 1033-1036.

13. Anandan SK, Ward JS, Brokx RD, Denny T, Bray MR, Patel DV, Xiao XY. Design and synthesis of thiazole-5hydroxamic acids as novel histone deacetylase inhibitors, Bioorg Chem Lett 2007; 17: 5995-5999.

14. Skehn P, Storeng R, Scudiero A, Monks J, Mcmohan D, Vistica D, Jonathan TW, Bokesch H. New Colorimetric 
Saha et al 